\title{
Processing Nannochloropsis gaditana biomass for the extraction of high value biocompounds
}

Rafael Sales², María del Carmen Cerón-García1, Elvira Navarro-López¹, Cynthia Victoria González-López¹, Mônica Yumi Tsuzuki², Francisco Gabriel Acién-Fernández¹, Francisco Javier Alarcón-López ${ }^{3}$, Emilio Molina-Grima ${ }^{1}$

${ }^{1}$ Department of Chemical Engineering and CIAIMBITAL, University of Almería, 04120, Almería- Spain

2 Department of Aquaculture, Federal University of Santa Catarina (UFSC), Florianópolis - Brazil

${ }^{3}$ Department of Biology and Geology, University of Almería, 04120, Almería - Spain

${ }^{*}$ Corresponding author:

E-mail address: mcceron@ual.es (María del Carmen Cerón García)

Address: Department of Chemical Engineering, University of Almería, Carretera Sacramento s/n. 04120, Almería, Spain

Telephone number: +34950015981

Fax: +34 950015484

\section{Abstract}

Extraction of carotenoids and fatty acids from microalgae is a technological bottleneck in processing. An improved extraction process was developed to scale the production of these bioproducts from Nannochloropsis gaditana. Different cell disruption methods were evaluated in terms of carotenoids release. Ethanol was substituted by isopropyl alcohol in a three-component solution of water:isopropyl alcohol:hexane (WIH), in which the extracts were separated by solution partitioning. This resulted in higher 
carotenoid and fatty acid recovery yields if compared to the standard method. The extraction method was replicated on a pilot scale obtaining similar carotenoid recovery yields, higher than those of the standard method. Although fatty acid recovery was lower than that of the small-scale tests, yields above $85 \%$ were obtained. This demonstrated that the method was scalable for the extraction of high-value products from microalgae up to $10 \mathrm{~L}$ reactor volume. The use of isopropyl alcohol, which is cheaper than ethanol, and the separation of the solution phases by partitioning (avoiding drying) could contribute to reduce operation costs of downstream processing.

\section{Keywords:}

Microalgae, Nannochloropsis gaditana, extraction, polyunsaturated fatty acids, carotenoids

\section{Introduction}

Microalgae produce a variety of high-value products, some of which are available commercially such as carotenoids, phycobilins, fatty acids, sterols, polyhydroxyalkonoates, and polysaccharides, which are used as nutraceuticals and functional foods for human and animal consumption (Borowitzka 2013).

Carotenoids are photosynthetic accessory pigments possessing several health benefits such as their antioxidant, anti-inflammatory and antitumor properties; they can also be used as natural food colorants (Sathasivam et al. 2017). The direct use of synthetic carotenoids for human consumption entails some health concerns due to their potential toxicity and its use is limited to animal feed, colorants and preservatives, while the natural ones have the advantage of being used as nutraceuticals (Gong and Bassi 2016). Consequently, the search for natural carotenoid sources has widened.

Similarly, there is great interest in the production of long-chain polyunsaturated fatty acids (LC-PUFAs) since they are responsible for regulating membrane fluidity, 
help to reduce the risk of heart disease, are essential for the structure of eye and brain tissue and are prostaglandin precursors (Sathasivam et al. 2017). Despite all the functions they are involved in, LC-PUFAs are not synthesized by humans or animals and therefore need to be obtained through the diet.

The resource-rich matrix of microalgae biomass provides a wide range of highvalue compounds ('t Lam et al. 2018); however, the downstream processing required to obtain them is expensive; for example, the extraction and purification of eicosapentaenoic acid (EPA) represents $57 \%$ of the total cost (Molina Grima et al. 2003). The use of the biorefinery concept, in which as many bioproducts as possible are obtained during processing, would provide extra income (Chew et al. 2017; 't Lam et al. 2018; Jacob-Lopes et al. 2019). On this basis, López-Rodríguez et al. (2019) proposed a multi-step extraction method for high-value added products from the dinoflagellate Amphidinium carterae which, in addition to the targeted product, enabled the recovery of amphidinolides and amphidinols (APDs) as well as carotenoids and fatty acids, thus contributing to the economic viability of the bioprocess.

The extraction of high-value products from microalgae using conventional atmospheric solid-liquid extraction methods has been extensively studied, and a wide range of solvents have been evaluated for this purpose. Furthermore, this method is inexpensive, does not require sophisticated equipment and is easy to scale up (Gong and Bassi 2016; Saini and Keum 2018). When choosing an extraction method, one should consider the chemical characteristics of the product, such as its polarity, the recovery yield of the desired bioproduct, the amount of solvent required, the extraction time and cost, the environmental impact and the method's scalability (Grosso et al. 2015).

The extraction method proposed by Cerón-García et al. (2018b, a) uses a three-component solution composed of water:ethanol:hexane (WEH) and $40 \% \mathrm{KOH}$ to extract the unsaponifiable lipid fraction (the carotenoids) and the saponified lipids (the 
fatty acids) from the microalgal biomass in a two-step procedure. Firstly, the fatty acids are saponified, then the solvents are evaporated, and the carotenoids are extracted with acetone and hexane. In the second step, the salts of fatty acids are released by $\mathrm{pH}$ adjustment and extracted with hexane. At the end, solvents are evaporated and no contamination of the final product should be checked by quality analysis. These solvents are amongst those listed as extraction solvents permitted for use in Europe (Directive 2009/32/CE and Directive 2010/59/UE) during the processing of raw materials, food-stuffs, food components or food ingredients.

One concern when using ethanol in a three-component solution is the price of the solvent. Other polar solvents can be used, such as isopropyl alcohol, which is cheaper than ethanol and has a lower polarity index; it can thus enhance recovery yields of non-polar carotenoids. Including isopropanol in the hexanic solution enhances the extraction of polar lipids that are bound to proteins from the microalgae cell membranes (Halim et al. 2011). Similarly, using isopropyl alcohol as a co-solvent for hexane also resulted in higher carotenoid recovery yields than when just using hexane (Ryckebosch et al. 2014).

The intermediate step of drying the solvents prior to extracting the carotenoids in the Cerón-García et al. (2018b) method is energy demanding and time consuming, which complicates its reproducibility at pilot scale. This requirement could be avoided by partitioning the solutions. Once the solvent proportions are adjusted, the hexanic non-polar phase with the carotenoids is separated from the hydroalcoholic polar phase, which contains the salts of fatty acids, using a separatory funnel to separate the two phases.

Microalgae from the genus Nannochloropsis have a resistant cell wall containing non-hydrolysable macromolecules called algaenans (Gelin et al. 1997). Any cell wall disruption procedure, whether mechanical, chemical, or enzymatic, can enhance the extraction of intracellular compounds such as carotenoids (Michelon et al. 
2012). The required volume of solvents for extracting the lipids is dependent on the solubility of the molecules as well as their capacity to penetrate the cell wall (Yao et al. 2012); hence, performing a cell breakage method might also be advantageous for reducing the amount of solvents.

The present study has improved the standard extraction method of CerónGarcía et al. (2018b) to obtain carotenoids and fatty acids from the microalga $N$. gaditana. This has made the procedure more feasible in terms of cost by using isopropyl alcohol instead of ethanol in the three-component solution and by replacing the drying step with solution partitioning. In addition, different biomass disruption methods were assessed to enhance the extraction of the biomolecules of interest.

\section{Materials and methods}

\subsection{Microalgal biomass}

Nannochloropsis gaditana strain B-3 was obtained from the Marine Culture Collection of the Institute of Marine Sciences of Andalucía (CSIC) Cádiz, Spain. This strain was cultured at the microalgae pilot plant of the University of Almería, Spain, in continuous mode at a $0.2 \mathrm{~d}^{-1}$ dilution rate in flat-panel and tubular photobioreactors (Menegol et al. 2019). When the cultures were at steady state, they were harvested. This resulted in two dry biomass batches, one during the spring (batch 1) and another during the summer (batch 2). The biomass was recovered at $9,000 \times \mathrm{g}$ for $5 \mathrm{~min}$ in a Sigma 4-15 centrifuge (Sartorius, Göttingen A.G., Germany), washed with $0.5 \mathrm{M}$ aqueous ammonium bicarbonate solution (Zhu and Lee 1997) and freeze-dried in a Cryodos 50 instrument (Telstar, Terrassa, Spain) prior to biochemical analyses.

\subsection{Cell disruption methods}


Different cell disruption methods were evaluated with the aim of increasing the extraction yields of the desired compounds. Compared to the control extraction without molturation treatment (WM), the biomass from batch 1 underwent five cell disruption methods: (i) mortar milling with alumina at a $1: 1(\mathrm{w} / \mathrm{w})$ biomass/alumina ratio at $25{ }^{\circ} \mathrm{C}$ (MA25), (ii) mortar milling with alumina at a $1: 1(\mathrm{w} / \mathrm{w})$ biomass/alumina ratio at $60{ }^{\circ} \mathrm{C}$ (MA60), (iii) ultrasounds (UTS), (iv) lab-scale bead milling (LBM), and (v) pilot-scale bead milling (PBM). The ultrasound equipment used was an Ultrason bath from JP Selecta (Barcelona, Spain). The LBM equipment was a Mini-Beadbeater-16 cell disrupter (Biospec Products Inc., Bartlesville, USA). The PBM equipment used $28 \mathrm{~mm}$ diameter beads. Each disruption method, and the WM control, were evaluated with 5 $\mathrm{mg}$ of dry biomass, using the analytical method to measure carotenoids described below. The carotenoid extraction attained for the sample without molturation treatment (WM, control) was stablished as $100 \%$ recovery yield. Therefore, if cell disruption methods improve carotenoids release, extraction yields above $100 \%$ would be achieved.

\subsection{Analytical procedures}

The carotenoids were analyzed on a Shimadzu SPDM10AV high performance liquid chromatograph (HPLC) (Shimadzu, Columbia, USA) using a photodiode array detector, applying the method adapted by Cerón-García et al. (2018a). The separation was performed on a LiChrospher@ 100 RP-18 column $(5-\mu \mathrm{m} ; 4.6 \times 150 \mathrm{~mm})$. The injection volume of each sample was $20 \mu \mathrm{L}$. Two eluents were used: (A) water:methanol 1:4 v/v; and (B) acetone:methanol 1:1 v/v in gradients of $25 \%$ B $0-8$ $\min , 75 \%$ B 8-18 min, 90\% B 18-23 min, 100\% B 25-27 min and 25\% B 27-32 min, at an elution rate of $1 \mathrm{~mL} / \mathrm{min}$, with absorbance detection at $360-700 \mathrm{~nm}$.

The fatty acid analysis was performed as described by Rodríguez-Ruiz et al. (1998). For the analysis of the initial biomass, $10 \mathrm{mg}$ of dry biomass was used. For the analysis of the microalga extracts, $1 \mathrm{~mL}$ of the extract was dried with argon $\left(25^{\circ} \mathrm{C}\right)$ and 
then processed in the same way. $1 \mathrm{~mL}$ of hexane was added to the samples, followed by $1 \mathrm{~mL}$ of the methylation mixture (methanol:acetyl chloride $20: 1 \mathrm{v} / \mathrm{v}$ ) and $50 \mu \mathrm{L}$ of an internal standard (25 mg nonadecanoic acid in $10 \mathrm{~mL}$ hexane) prior to heating at 105 ${ }^{\circ} \mathrm{C}$ for 20 minutes. After cooling down, $1 \mathrm{~mL}$ of water was added so that the phases could be separated by centrifugation. The hexanic (upper) phase, containing the fatty acid methyl esters (FAMEs), obtained from saponifiable lipids present in microalgal biomass, was analyzed with an Agilent Technologies 6890N gas chromatograph (Avondale, PA, USA). The measurements were carried out in duplicate.

The dry biomass was also fractioned using Sep-pack plus AT020520 single-use silica gel cartridges (Waters Corporation, Milford, MA, USA). Saponifiable lipids were separated into three fractions with different polarities, neutral lipids, glycolipids and phospholipids. A $10 \mathrm{mg}$ sample of dry biomass was added to a glass Pyrex tube and dissolved with $0.5 \mathrm{~mL}$ of chloroform; the concentrated chloroform phase of the lipids was then introduced into the cartridge. A further $30 \mathrm{~mL}$ of chloroform was passed through the cartridge with the eluent containing the neutral lipids. Subsequently, $30 \mathrm{~mL}$ of acetone and $20 \mathrm{~mL}$ of chloroform:methanol $(85: 15 \mathrm{v} / \mathrm{v})$ were passed through the cartridge to collect the glycolipids. Finally, $30 \mathrm{~mL}$ of methanol was passed through the cartridge to recover the phospholipid fraction. All eluents were evaporated in a R210 rotary evaporator with a V-700 vacuum pump and a V-850 controller (Buchi, Flawil, Switzerland), and the content of each fraction was converted to FAMEs by methylation and then analyzed by gas chromatography as described by Navarro López et al. (2016). All analytical measurements were performed in triplicate.

\subsection{Multi-step approach for the small-scale extraction of carotenoids and fatty acids}

The biomass with a higher content of carotenoids (batch 1) was used for the small-scale experiment. Prior to extraction, the biomass was milled in a mortar with 
alumina $(1: 1 \mathrm{w} / \mathrm{w})$. Modifications were made to the extraction method proposed by Cerón-García et al. (2018b). The substitution of ethanol with alcohol isopropyl in the three-component solution was evaluated, the hexanic phase (with the unsaponified lipids) being separated from the hydroalcoholic phase (with the fatty acid salts) by partitioning.

The carotenoid and fatty acid extraction was compared using three-component solutions of different compositions. Water-ethanol-hexane (6:77:17) and $40 \% \mathrm{KOH}$ d.w. (WEH) was compared to water-isopropyl-hexane and $40 \% \mathrm{KOH}$ d.w. (WIH) with waterisopropyl-hexane proportions of $6: 77: 17,9: 74: 17,10: 73: 17$ and $12: 71: 17$. Then, the phases of the solutions were separated using either water-ethanol-hexane or waterisopropyl alcohol-hexane at the ratios of $36: 14: 50$ and 10:40:50, respectively.

The first step (saponification) was performed under the best disruption conditions (maceration in a water-bath at $60^{\circ} \mathrm{C}$ ) for $2 \mathrm{~min}$. The monophasic solution was partitioned by adding water and hexane, then separated by centrifugation. After the separation, the upper phase containing the unsaponified lipids and the lower phase containing the saponifiable lipids were collected to analyze the carotenoid and fatty acid compositions, respectively. Recovery yields were calculated as the percentage of carotenoid and fatty acid contents with respect to the total carotenoids and fatty acids in the original biomass, extracted by the standard method.

\subsection{Scaled-up multi-step approach for the extraction of carotenoids and fatty acids}

The biomass from batches 1 and 2 were used to scale-up the improved carotenoid and fatty acid extraction method (Fig. 1). Prior to the extraction, the biomass was milled in the PBM for 10 min. The three-component WIH 12:71:17 solution (with $40 \% \mathrm{KOH}$ ) and the WIH 10:40:50 solution were tested. The saponification reaction was performed using a $10-\mathrm{L}$ reactor jacketed to maintain $60^{\circ} \mathrm{C}$ and supplying agitation at 
$150 \mathrm{rpm}$ for $1 \mathrm{~h}$. The volume of solvents added to the biomass was calculated based on the ratio volume for saponifiable lipids proposed by Hita Peña et al. (2015), such that

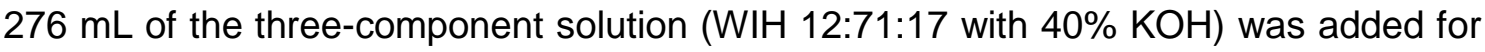
$1 \mathrm{~g}$ of saponifiable lipids from the dried $N$. gaditana biomass. Consequently, considering the $14 \%$ of saponifiable lipids for the batches, $4830 \mathrm{~mL}$ of the three-component solution (WIH in a ratio of $580: 3429: 821$ ) was added to $125 \mathrm{~g}$ of biomass. The extract was filtered and the residual biomass was washed with $400 \mathrm{~mL}$ WIH solution $\left(3.2 \mathrm{~mL} \mathrm{~g}^{-1}\right.$ dry weight) without $\mathrm{KOH}$. For the extraction of carotenoids, the solvent ratio was adjusted to $10: 40: 50 \mathrm{WIH}$, by adding 278 and $3465 \mathrm{~mL}$ of water and hexane, respectively, and agitating for $30 \mathrm{~min}$ at $100 \mathrm{rpm}$. The phases were allowed to separate over $30 \mathrm{~min}$. The hydroalcoholic phase, which contained the salts of fatty acids, had its $\mathrm{pH}$ adjusted to 2.0. Water and hexane were then added to the hydroalcoholic phase in a ratio of 0.6:0.3:1 in accordance with Navarro López et al. (2016). Considering a volume of approximately $4 \mathrm{~L}$ for the hydroalcoholic extract obtained from the first step, 2.4 and 1.2 $L$ of water and hexane were added, respectively. The second reaction lasted $10 \mathrm{~min}$ at $150 \mathrm{rpm}$, and the phases were allowed to separate for $30 \mathrm{~min}$, this process being repeated twice. Samples were taken to evaluate the carotenoid and fatty acid composition and recovery yields at several steps: saponification and washing of the biomass (SAP), extraction (EXT), and the residual biomass (RES). The extraction

procedure was repeated two times for each batch. The recovery yields were determined as the content of carotenoids and fatty acids in the extraction steps with respect to the content of carotenoids and fatty acids in the original biomass (extracted by the standard method.

\subsection{Statistical analysis}

Results are expressed as mean \pm standard deviation. Data in percentages were arcsine square root transformed and checked for homogeneity and normality, with the 
results being compared by one-way analysis of variance (ANOVA). In addition, significantly different treatments $(p<0.05)$ were determined using Tukey's post-hoc test.

\section{Results and discussion}

In order to scale up the extraction processes, small-scale studies are essential to obtain data on the solvent phase equilibrium, the mass transfer rate and the solubility (Grosso et al. 2015). Some algal compounds are not easily reached by solvents; therefore, the biomass must be submitted to some form of cell disruption before extraction, with the industrial applicability of such procedures being based on scalability, energy requirements, accessibility to the desired compounds and mass transfer (Roux et al. 2017). Accordingly, in the present study, the proposed improvements to the standard extraction method were tested on the small-scale prior to their pilot-scale application.

\subsection{Optimization of extraction processes}

The carotenoid and fatty acid profiles of batches 1 and 2 are presented in Tables 1 and 2, respectively. The polar fraction (glycolipids and phospholipids) and the non-polar fraction of the lipids from both batches were $65 \% \pm 1.08$ and $34 \% \pm 0.58$ for batch 1 , and $29 \% \pm 0.72$ and $71 \% \pm 0.68$ for batch 2 , respectively.

The results from the disruption methods evaluated are presented in Table 3. The carotenoid concentrations in the samples submitted to the MA25, MA60, UTS, LBM and PBM disruption methods were similar and significantly higher than the sample extracted without any pre-treatment $(\mathrm{WM} ; \mathrm{p}<0.05)$. The application of cell disruption methods was recommended to improve the recovery of intracellular products from the microalga, such as pigments, fatty acids and proteins (Molina Grima et al. 2003; Vizcaíno et al. 2019). Microalgae from the genus Nannochloropsis have robust cell 
walls made up of algaenan and cellulose, which are resistant to hydrolysis (Scholz et al. 2014); therefore, it is necessary to break up these cell walls using mechanical disruption methods.

The total carotenoid recovery yields from the different disruption methods are presented in Fig. 2. The control sample (WM, no cell disruption) was stablished as $100 \%$ recovery yield. The recovery yields of the samples submitted to disruption methods were significantly higher than for the biomass that did not undergo molturation (WM; $p<0.05)$; nonetheless, no differences were observed between the different disruption methods themselves $(p>0.05)$. On reviewing the various cell disruption methods, Gong and Bassi (2016) classified grinding as efficient but time-consuming, which restricts its scalability. Ultrasound was classified as highly efficient although different studies have shown variable results. Bead milling, on the other hand, was classified as highly efficient but concerns were raised relating to small-sized cells. Although $N$. gaditana is a small-cell organism $(\sim 2 \mu \mathrm{m})$, bead milling was efficient in disrupting the cells. Therefore, results show that there is a need to use a cell disruption method and among them bead milling could be the preferred one because of the easiness to be used in an industrial scaled-up process. However, any of the other tested methods could be chosen for a pilot-scale extraction of high-value products.

\subsection{Multi-step approach for the small-scale extraction of carotenoids and fatty acids}

The carotenoid and fatty acid composition from the small-scale extraction with the three-component solution (6:77:17) using ethanol and isopropyl alcohol are presented in Tables 4 and 5, respectively. The total concentration of carotenoids and fatty acids extracted with the isopropyl alcohol three-component solution (WIH) was higher than with ethanol (WEH). The polarity index of isopropyl alcohol is lower (4.3) than ethanol (5.2), which is likely to have enhanced the extraction of non-polar lipids. 
Regarding the partitioning ratios, 10:40:50 presented a higher concentration of carotenoids than 36:14:50, regardless of which alcohol was used (ethanol or isopropyl alcohol). However, it was not possible to measure the fatty acid concentration of the samples extracted with the $36: 14: 50$ ratio because of its elevated water content.

The $40 \% \mathrm{KOH}$ in the three-component solution was not well dissolved in the 6:77:17 solution ratio using isopropyl alcohol. For this reason, other solvent ratios with higher water contents were evaluated, namely 9:74:17, 10:73:17 and 12:71:17. The carotenoid and fatty acid composition from the samples extracted using these different three-component solutions $(\mathrm{WIH})$ are presented in Tables 4 and 5 , respectively. Overall, the different solvent ratios presented similar concentrations of carotenoids and fatty acids. Furthermore, the carotenoid concentration from the samples extracted with the $10: 40: 50$ WIH partitioning ratio was higher than for the $36: 14: 50$ WIH solution ratio. The carotenoid content in the 12:71:17 WIH treatment with the 10:40:50 WIH partitioning ratio was $2.05 \%$, while the carotenoid content of the same solution, but with the $36: 14: 50$ WIH partitioning ratio, was less at $1.06 \%$. The fatty acid concentration was only evaluated for the 10:40:50 WIH ratio (Table 5).

The carotenoid recovery yields from the small-scale extraction are presented in Fig. 3. When comparing the use of ethanol or isopropyl alcohol in the three-component solutions, at both proportions (6:36 and 6:10), the recovery yield with isopropyl alcohol was significantly higher than when using ethanol $(p<0.05)$. Using solvents with different polarities facilitates the extraction of complex molecules that have diverse polarities. Carotenoids are composed of a complex mixture of non-polar compounds, such as carotenes (lycopene and $\beta$-carotene), and xanthophylls, which contain polar groups (violaxanthin, zeaxanthin and canthaxanthin). Thus, to simultaneously extract both types of carotenoids, a mixture of polar and non-polar solvents are used.

The WIH 6:10 treatment presented higher carotenoid recovery yields than the WEH 6:36, WIH 6:36 and WEH 6:10 treatments $(p<0.05)$. The WIH 9:10, 10:10 and 
12:10 treatments showed the highest carotenoid recovery yields, with the WIH 10:10 and 12:10 treatments being significantly higher than for WIH 6:36, WIH 10:36 and WIH 12:36 $(p<0.05)$. The higher yield treatments were those with a higher concentration of isopropyl alcohol, as this aided the extraction. The carotenoid recovery yields of the treatments WIH 9:10, 10:10 and 12:10 were higher than 100\% (which corresponds to the control, measured with the standard method that uses ethanol) so the proposed method using isopropyl alcohol improved carotenoid extraction.

Carotenes are found in free form while xanthophylls are normally coupled to fatty acids as esters; hence, when extracting carotenoids, a saponification step is essential for releasing the esterified xanthophylls in free form (Mercadante et al. 2017). Probably, the saponification process in the 6:77:17 WIH monophasic solution did not perform as efficiently as in the solutions with different ratios; thus, some of the xanthophylls might have remained esterified. In addition, other compounds may have interfered with the analysis, such as triacylglycerides (TAGs), proteins, lipids, chlorophylls and carbohydrates (Mercadante et al. 2017; Saini and Keum 2018), resulting in lower recovery yields.

The recovery yields of fatty acids in the small-scale extraction are presented in Fig. 4. The fatty acid recovery yields for both solutions WEH 6:10 and WIH 6:10 were similar $(p>0.05)$. The WIH 9:10, 10:10 and 12:10 treatments presented higher recovery yields, while the $\mathrm{WIH} \mathrm{10:10} \mathrm{and} \mathrm{WIH} 12: 10$ treatments were significantly higher than for WEH 6:10 and WIH 6:10 ( $p<0.05)$. Similarly to the extraction of carotenoids, fatty acid recovery yields of the treatments WIH 10:10 and WIH 12:10 were higher than $100 \%$ showing an extraction improvement by the use of isopropyl alcohol.

The 12:71:17 WIH saponification solution with $40 \% \mathrm{KOH}$ and the 10:40:50 WIH partitioning ratio presented the best results for carotenoid and fatty acid extraction; hence, these solution ratios were chosen for replication in the pilot-scale experiments. 


\subsection{Scaled-up multi-step approach for the extraction of carotenoids and fatty acids}

The carotenoid profiles from batches 1 and 2 were very different from each other, with the total carotenoid content of batch 1 being much higher. Even so, the carotenoid profiles were similar. The most abundant carotenoids in both batches were violaxanthin and $\beta$-carotene. The fatty acid content and profile from batch 1 was quite different from batch 2. Batch 1 showed higher LC-PUFAs while batch 2 had higher saturated, monounsaturated and total fatty acids. The fraction of polar and non-polar lipids was also different, with batch 1 having more polar lipids and batch 2 more nonpolar lipids.

The carotenoid and fatty acid recovery yields from the scaled-up experiment are presented in Figs. 5 and 6 . The carotenoid recovery yield patterns when extracting both batches were similar; that is to say, the total carotenoid recovery yields from samples having undergone the saponification step in the pilot-scale procedure $\left(\mathrm{Y}_{\text {sap }}\right)$ were between $130-160 \%$, and were similar to the extraction step $\left(Y_{\text {ext }} ; p>0.05\right)$. There were almost no carotenoids left in the residual biomass after saponification, with $Y_{\text {res }}$ being lower than 8\%, a value significantly lower than that of $Y_{\text {sap }}$ and $Y_{\text {ext }}(p<0.05)$. The total recovery yields for the saponification and the extraction steps performed on the pilotscale were similar to the results obtained on the small scale for the WIH 12:10 treatment $(p=0.684)$. These results indicate that the scaling-up of the saponification and carotenoid extraction steps was successful, with all the compounds present in the saponified extract being successfully recovered and the residual biomass showing few carotenoids. Comparing the two batches, batch 1 had higher carotenoid recovery yields for the saponification and extraction steps than batch $2(p<0.05)$; nevertheless, the residual yield was similar. The higher carotenoid recovery yield in batch 1 was probably due to a higher $\beta$-carotene content in the biomass than in batch 2 (33\% compared to 
26\%). Since $\beta$-carotene had non-polar characteristics, it had a higher affinity for isopropyl alcohol and was extracted more efficiently.

The fatty acid $Y_{\text {sap }}$ was high, at around $100 \%$ for both batches. The $Y_{\text {ext }}$ for batch 1 and 2 were around $90 \%$ and $75 \%$, respectively, both significantly lower than the $Y_{\text {sap }}$ ( $p<0.05)$. Possibly, the amount of fatty acids remaining (25\%) was not fully extracted from the hydroalcoholic phase. In the present study, two series of extractions were performed. Perhaps a third one would have enhanced the extraction step recovery yield. The fatty acid recovery yield from the residual biomass was low, at around $2 \%$. The extraction of fatty acids on the small scale (treatment WIH 12:10) was significantly higher than both extract samples $\left(Y_{\text {sap }}\right.$ and $\left.Y_{\text {ext }}\right)$ in the pilot scale extraction $(p<0.05)$. Several parameters affected the extraction kinetics, such as the amount of lipids in the biomass, the reaction time and the mass transfer coefficient, which was related to the degree of solution agitation, the ratio of organic solvent to microalgae biomass and the temperature (Halim et al. 2012). The mass transfer of the extraction changes from the small-scale to the pilot scale situation, affecting the interaction of solvents with the substrate. Possibly for this reason, some of the fatty acids from the biomass were not fully extracted in the pilot scale experiments, causing fatty acid recovery yields to be lower than for the small-scale extractions. Therefore, attention must be paid to the mechanism of lipid mass transfer from the microalgae biomass to the solvents in the scaled-up extractions. The recovery yields from the saponification step and the residual biomass were similar for both batches; however, the fatty acid recovery yield ( $\left.Y_{\text {ext }}\right)$ from batch 1 was significantly higher than from batch 2 . It was hypothesized that including isopropyl alcohol in the three-component solution (with hexane, a non-polar solvent) would enhance the extraction of polar lipids (phospholipids, glycolipids, and cholesterols). Since batch 1 had a higher level of polar lipids than batch 2 , the biochemical composition of the biomass would have been a positive influence on the extraction of the fatty acids. 


\section{Conclusions}

The extraction of high-value bioproducts from N. gaditana has been improved. It is necessary to pretreat the biomass in order to release intracellular compounds. Bead milling is proposed as the most adequate method for a scaled-up process. The extraction step, which employed isopropanol instead of ethanol in the three-component extraction solution, resulted in a similar recovery yield of fatty acids $(85 \%)$, but higher yields of carotenoids recovery $(120 \%)$. The lower price of isopropanol compared to ethanol and the labour-saving partitioning of the three-component solution could contribute to reduce operation costs in the downstream processing.

\section{Acknowledgements}

This research was supported by the European Union's Horizon 2020 Research and Innovation Programme under Grant Agreement No. 727874 SABANA. The study was financed in part by the Coordenação de Aperfeiçoamento de Pessoal de Nível Superior - Brasil (CAPES) - Finance Code 001. M.Y. Tsuzuki was granted a research fellowship from the National Council for Scientific and Technological Development (CNPq 306078/2017-1).

\section{Declarations of interest}

The authors declare that they have no conflicts of interest.

\section{References}

't Lam GP, Vermuë MH, Eppink MHM, et al (2018) Multi-product microalgae biorefineries: from concept towards reality. Trends Biotechnol 36:216-227. doi: 10.1016/j.tibtech.2017.10.011

Borowitzka MA (2013) High-value products from microalgae-their development and commercialisation. J Appl Phycol 25:743-756. doi: 10.1007/s10811-013-9983-9 
Cerón-García MC, González-López CV, Camacho-Rodríguez J, et al (2018a) Maximizing carotenoid extraction from microalgae used as food additives and determined by liquid chromatography (HPLC). Food Chem 257:316-324. doi: 10.1016/j.foodchem.2018.02.154

Cerón-García MC, González-López CV, Fernandez-Sevilla JM, Molina Grima E (2018b) Preparative recovery of carotenoids from microalgal biomass. In: Editorial Methods Molecular Biology, 1852nd edn. Springer Nature Experiments, pp 107-115

Chew KW, Yap JY, Show PL, et al (2017) Microalgae biorefinery: High value products perspectives. Bioresour Technol 229:53-62. doi: 10.1016/j.biortech.2017.01.006

Gelin F, Boogers I, Noordeloos AAM, et al (1997) Resistant biomacromolecules in marine microalgae of the classes Eustigmatophyceae and Chlorophyceae: Geochemical implications. Org Geochem 26:659-675. doi: 10.1016/S01466380(97)00035-1

Gong M, Bassi A (2016) Carotenoids from microalgae: A review of recent developments. Biotechnol Adv 34:1396-1412. doi: 10.1016/j.biotechadv.2016.10.005

Grosso C, Valentão P, Ferreres F, Andrade PB (2015) Alternative and efficient extraction methods for marine-derived compounds. Mar Drugs 13:3182-3230. doi: $10.3390 / \mathrm{md} 13053182$

Halim R, Danquah MK, Webley PA (2012) Extraction of oil from microalgae for biodiesel production: A review. Biotechnol Adv 30:709-732. doi: 10.1016/j.biotechadv.2012.01.001

Halim R, Gladman B, Danquah MK, Webley PA (2011) Oil extraction from microalgae for biodiesel production. Bioresour Technol 102:178-185. doi: 10.1016/j.biortech.2010.06.136

Jacob-Lopes E, Maroneze MM, Deprá MC, et al (2019) Bioactive food compounds from microalgae: an innovative framework on industrial biorefineries. Curr Opin Food Sci 25:1-7. doi: 10.1016/j.cofs.2018.12.003

López-Rodríguez M, Cerón-García MC, López-Rosales L, et al (2019) Assessment of multi-step processes for an integral use of the biomass of the marine microalga Amphidinium carterae. Bioresour Technol 282:370-377. doi: 10.1016/j.biortech.2019.03.041

Menegol T, Romero-Villegas Gl, López-Rodríguez M, et al (2019) Mixotrophic production of polyunsaturated fatty acids and carotenoids by the microalga Nannochloropsis gaditana. J Appl Phycol 31:2823-2832. doi: 10.1007/s10811-01901828-3

Mercadante AZ, Rodrigues DB, Petry FC, Mariutti LRB (2017) Carotenoid esters in foods - A review and practical directions on analysis and occurrence. Food Res Int 99:830-850. doi: 10.1016/j.foodres.2016.12.018

Michelon M, de Matos de Borba T, da Silva Rafael R, et al (2012) Extraction of carotenoids from Phaffia rhodozyma: A comparison between different techniques of cell disruption. Food Sci Biotechnol 21:1-8. doi: 10.1007/s10068-012-0001-9 
Molina Grima E, Belarbi EH, Acién Fernández FG, et al (2003) Recovery of microalgal biomass and metabolites: Process options and economics. Biotechnol Adv 20:491515. doi: 10.1016/S0734-9750(02)00050-2

Navarro López E, Robles Medina A, González Moreno PA, et al (2016) Extraction of microalgal lipids and the influence of polar lipids on biodiesel production by lipasecatalyzed transesterification. Bioresour Technol 216:904-913. doi: 10.1016/j.biortech.2016.06.035

Rodríguez-Ruiz J, Belarbi E-H, Sánchez JLG, Alonso DL (1998) Rapid simultaneous lipid extraction and transesterification for fatty acid analyses. Biotechnol Tech 12:689691. doi: 10.1023/A:1008812904017

Roux J, Lamotte H, Achard J (2017) An overview of microalgae lipid extraction in a biorefinery framework. Energy Procedia 112:680-688. doi: 10.1016/j.egypro.2017.03.1137

Ryckebosch E, Bruneel C, Termote-Verhalle R, et al (2014) Influence of extraction solvent system on extractability of lipid components from different microalgae species. Algal Res 3:36-43. doi: 10.1016/j.algal.2013.11.001

Saini RK, Keum YS (2018) Carotenoid extraction methods: A review of recent developments. Food Chem 240:90-103. doi: 10.1016/j.foodchem.2017.07.099

Sathasivam R, Radhakrishnan R, Hashem A, Abd_Allah EF (2017) Microalgae metabolites: A rich source for food and medicine. Saudi J Biol Sci 26:709-722. doi: 10.1016/j.sjbs.2017.11.003

Scholz MJ, Weiss TL, Jinkerson RE, et al (2014) Ultrastructure and composition of the Nannochloropsis gaditana cell wall. Eukaryot Cell 13:1450-1464. doi: 10.1128/EC.00183-14.

Vizcaíno AJ, Sáez MI, Martínez TF, et al (2019) Differential hydrolysis of proteins of four microalgae by the digestive enzymes of gilthead sea bream and Senegalese sole. Algal Res 37:145-153. doi: 10.1016/j.algal.2018.11.018

Yao L, Gerde JA, Wang T (2012) Oil extraction from microalga Nannochloropsis sp. with isopropyl alcohol. J Am Oil Chem Soc 89:2279-2287. doi: 10.1007/s11746-0122124-9

Zhu CJ, Lee YK (1997) Determination of biomass dry weight of marine microalgae. J Appl Phycol 9:189-194. doi: 10.1023/A:1007914806640 


\section{Figure Captions}

Fig. 1 Scheme of the extraction process for recovering carotenoids and fatty acids simultaneously from the Nannochloropsis gaditana microalga, a modification of the method proposed by Cerón-García et al. 2018b.

Fig. 2 Carotenoid recovery yields (\%) of Nannochloropsis gaditana batch 1 submitted to different disruption methods: i) without molturation (WM), ii) mortar milling with alumina at a $1: 1 \mathrm{w} / \mathrm{w}$ biomass/alumina ratio at 25ㅇ (MA25); (iii) mortar milling with alumina at a $1: 1 \mathrm{w} / \mathrm{w}$ biomass/alumina ratio at $60^{\circ} \mathrm{C}$ (MA60); (iv) ultrasound (UTS); (v) lab bead mill (LBM); (vi) pilot bead mill (PBM). Results are presented as the average \pm SD of two independent experiments. * Extraction recovery yield (\% d.w.): percentage of carotenoids extracted with respect to the compounds present in the biomass without any molturation (batch 1) extracted by the standard method (Cerón-García et al. 2018b). Different letters represent a significant difference between treatments.

Fig. 3 Carotenoid recovery yields (\%) of Nannochloropsis gaditana biomass batch 1 in the small-scale experiment for the treatments WEH 6:36 - ethanol solution, monophasic - 6:77:17 biphasic 36:14:50; WIH 6:36 - isopropyl alcohol solution, monophasic - 6:77:17 biphasic 36:14:50; WEH 6:10 - ethanol solution, monophasic 6:77:17 biphasic - 10:40:50; WIH 6:10 - isopropyl alcohol solution, monophasic 6:77:17 biphasic - 10:40:50; WIH 9:36 - isopropyl alcohol solution, monophasic 9:74:17 biphasic - 36:14:50; WIH 10:36 - isopropyl alcohol solution, monophasic 10:73:17 biphasic - 36:14:50; WIH 12:36 - isopropyl alcohol solution, monophasic 12:71:17 biphasic - 36:14:50; WIH 9:10 - isopropyl alcohol solution, monophasic 9:74:17 biphasic - 10:40:50; WIH 10:10 - isopropyl alcohol solution, monophasic 10:73:17 biphasic - 10:40:50; WIH 12:10 - isopropyl alcohol solution, monophasic 12:71:17 biphasic $-10: 40: 50$. Results are presented as the average $\pm \operatorname{SD}(n=3)$. * Extraction recovery yield (\% d.w.): percentage of carotenoids extracted with respect to the compounds present in the initial biomass (batch 1) extracted by the standard 
method (Cerón-García et al. 2018b). Different letters represent a significant difference between treatments, lowercase letters represent the comparison of the threecomponent solutions (WEH 6:36, WEH 6:10, WIH 6:30 and WIH 6:10); uppercase letters represent the comparison between the three-component solution with isopropyl alcohol.

Fig. 4 Fatty acid recovery yields (\%) of Nannochloropsis gaditana batch 1 in the smallscale experiment for the treatments: WEH 6:10 - ethanol solution, monophasic 6:77:17 biphasic - 10:40:50; WIH 6:10 - isopropyl alcohol solution, monophasic 6:77:17 biphasic - 10:40:50; WIH 9:10 - isopropyl alcohol solution, monophasic 9:74:17 biphasic - 10:40:50; WIH 10:10 - isopropyl alcohol solution, monophasic 10:73:17 biphasic - 10:40:50; WIH 12:10 - isopropyl alcohol solution, monophasic 12:71:17 biphasic $-10: 40: 50$. Results are presented as the average \pm SD $(n=3)$. * Extraction recovery yield (\% d.w.): percentage of fatty acids extracted with respect to the compounds present in the initial biomass (batch 1).

Fig. 5 Carotenoid recovery yields (\%) of Nannochloropsis gaditana biomass (batches 1 and 2) extracted by the improved method at the pilot scale after saponification (SAP); the extract fraction (EXT) and for the residual algal biomass (RES). Results are presented as the average $\pm S D$ of two independent experiments. * Extraction recovery yield (\% d.w.): percentage of carotenoids extracted with respect to the compounds present in the initial biomass (batches 1 and 2) extracted by the standard method (Cerón-García et al. 2018b) for the saponification step, the extraction and the residue.

Fig. 6 Fatty acid recovery yield (\%) of Nannochloropsis gaditana biomass (batches 1 and 2) extracted by the improved method at the pilot scale for the extract after saponification (SAP); the extract fraction (EXT) and for the residual algal biomass (RES). Results are presented as the average \pm SD of two independent experiments. ${ }^{*}$ Extraction recovery yield (\% d.w.): percentage of fatty acids extracted with respect to 
the compounds present in the initial biomass (batches 1 and 2) for the saponification step, the extraction and the residue. 
Table 1 - Carotenoid content in dry weight (\% d.w.) mean \pm SD of batches 1 (spring) and 2 (summer) of Nannochloropsis gaditana biomass extracted by the standard method (Cerón-García et al. 2018b).

\begin{tabular}{lll}
\hline Carotenoid & \multicolumn{1}{l}{$\begin{array}{l}\text { \% d.w. } \\
\text { Batch 1 }\end{array}$} & Batch 2 \\
\hline Neoxanthin & $0.066 \pm 0.009$ & $0.054 \pm 0.006$ \\
Violaxanthin & $0.899 \pm 0.064$ & $0.123 \pm 0.013$ \\
Anteroxanthin & $0.058 \pm 0.046$ & $0.005 \pm 0.001$ \\
Vaucheroxanthin & $0.077 \pm 0.0750 .015 \pm 0.002$ \\
Zeaxanthin & $0.074 \pm 0.0610 .015 \pm 0.001$ \\
Vaucheroxanthin ester $0.044 \pm 0.0540 .001 \pm 0.001$ \\
Canthaxanthin & $0.009 \pm 0.0070 .003 \pm 0.000$ \\
B-carotene & $0.622 \pm 0.0390 .077 \pm 0.012$ \\
Total & $1.850 \pm 0.2170 .292 \pm 0.036$ \\
\hline
\end{tabular}


Table 2 - Fatty acid content in dry weight (\% d.w.) mean \pm SD of batches 1 (spring) and 2 (summer) of Nannochloropsis gaditana biomass.

\begin{tabular}{lllll}
\hline Fatty acid & \multicolumn{2}{l}{$\begin{array}{l}\text { \% d.w. } \\
\text { Batch } 1\end{array}$} & \multicolumn{3}{l}{ Batch 2} \\
\hline $14: 0$ & 0.78 & \pm 0.04 & 1.75 & \pm 0.00 \\
$16: 0$ & 1.90 & \pm 0.07 & 7.33 & \pm 0.07 \\
$16: 1 \mathrm{n} 7$ & 2.40 & \pm 0.07 & 5.32 & \pm 0.04 \\
$18: 0$ & & & 0.18 & \pm 0.00 \\
$18: 1 \mathrm{n} 9$ & 0.53 & \pm 0.03 & 1.80 & \pm 0.02 \\
$18: 2 \mathrm{n} 6$ & 0.45 & \pm 0.01 & 0.30 & \pm 0.00 \\
$20: 4 \mathrm{n} 6$ & 1.10 & \pm 0.11 & 0.77 & \pm 0.00 \\
$20: 5 \mathrm{n} 3$ & 5.62 & \pm 0.06 & 2.31 & \pm 0.03 \\
Others & 1.56 & \pm 0.15 & 0.16 & \pm 0.02 \\
$\sum$ SFA & 2.69 & \pm 0.05 & 9.26 & \pm 0.07 \\
$\sum$ MUFA & 2.93 & \pm 0.10 & 7.12 & \pm 0.06 \\
$\sum$ LC-PUFA & 6.72 & \pm 0.14 & 3.08 & \pm 0.03 \\
Total FA & 14.37 & \pm 0.37 & 19.92 & \pm 0.14 \\
\hline
\end{tabular}


Table 3 - Carotenoid content in dry weight (\% d.w.) mean \pm SD from Nannochloropsis gaditana batch 1 submitted to different disruption methods: without maceration (WM); mortar with alumina at a biomass/alumina ratio 1:1 w/w at 25으 (MA25); mortar with alumina at a biomass/alumina ratio $1: 1 \mathrm{w} / \mathrm{w}$ at $60^{\circ} \mathrm{C}$ (MA60); ultrasound (UTS); lab bead mill (LBM); pilot bead mill (PBM). Different letters represent significant difference between treatments $(p<0.05)$.

\begin{tabular}{|c|c|c|c|c|}
\hline \multirow{2}{*}{ Carotenoid } & \multicolumn{4}{|l|}{$\%$ d.w. } \\
\hline & MA25 & MA60 & LBM & PBM \\
\hline Neoxanthin & \multicolumn{4}{|c|}{$0.033 \pm 0.002^{b} 0.057 \pm 0.003^{a} 0.070 \pm 0.003^{a} 0.059 \pm 0.003^{a} 0.063 \pm 0.003^{a} 0.066 \pm 0.003^{a}$} \\
\hline /iolaxanthin & \multicolumn{4}{|c|}{$0.450 \pm 0.022^{b} 0.776 \pm 0.039^{a} 0.953 \pm 0.048^{a} 0.810 \pm 0.041^{a} 0.864 \pm 0.043^{a} 0.899 \pm 0.045^{a}$} \\
\hline xanthin & \multicolumn{4}{|c|}{$0.029 \pm 0.001^{b} 0.050 \pm 0.003^{a} 0.061 \pm 0.003^{a} 0.052 \pm 0.003^{a} 0.056 \pm 0.003^{a} 0.058 \pm 0.003^{a}$} \\
\hline auchorovanthi & \multicolumn{4}{|c|}{$0.039 \pm 0.002^{b} 0.067 \pm 0.003^{a} 0.082 \pm 0.004^{a} 0.070 \pm 0.003^{a} 0.074 \pm 0.004^{a} 0.077 \pm 0.004^{a}$} \\
\hline Zeaxanthin & \multicolumn{4}{|c|}{$0.037 \pm 0.002^{b} 0.064 \pm 0.003^{a} 0.078 \pm 0.004^{a} 0.067 \pm 0.003^{a} 0.071 \pm 0.004^{a} 0.074 \pm 0.004^{a}$} \\
\hline \multicolumn{5}{|c|}{ Vaucheroxanthin ester $0.022 \pm 0.001^{\mathrm{b}} 0.038 \pm 0.002^{\mathrm{a}} 0.047 \pm 0.002^{\mathrm{a}} 0.040 \pm 0.002^{\mathrm{a}} 0.042 \pm 0.002^{\mathrm{a}} 0.044 \pm 0.002^{\mathrm{a}}$} \\
\hline Cantaxanthin & \multicolumn{4}{|c|}{$0.005 \pm 0.000^{b} 0.008 \pm 0.000^{a} 0.010 \pm 0.000^{a} 0.008 \pm 0.000^{a} 0.009 \pm 0.000^{a} 0.009 \pm 0.000^{a}$} \\
\hline carotene & \multicolumn{4}{|c|}{$0.311 \pm 0.016^{b} 0.537 \pm 0.027^{a} 0.659 \pm 0.033^{a} 0.560 \pm 0.028^{a} 0.598 \pm 0.030^{a} 0.622 \pm 0.031^{a}$} \\
\hline Total & \multicolumn{4}{|c|}{$0.925 \pm 0.046^{b} 1.596 \pm 0.080^{a} 1.960 \pm 0.098^{a} 1.666 \pm 0.083^{a} 1.778 \pm 0.089^{a} 1.850 \pm 0.092^{a}$} \\
\hline
\end{tabular}


Table 4 - Carotenoid content in dry weight (\% d.w.) mean \pm SD of carotenoid composition of Nannochloropsis gaditana batch 1 extracted in small scale.

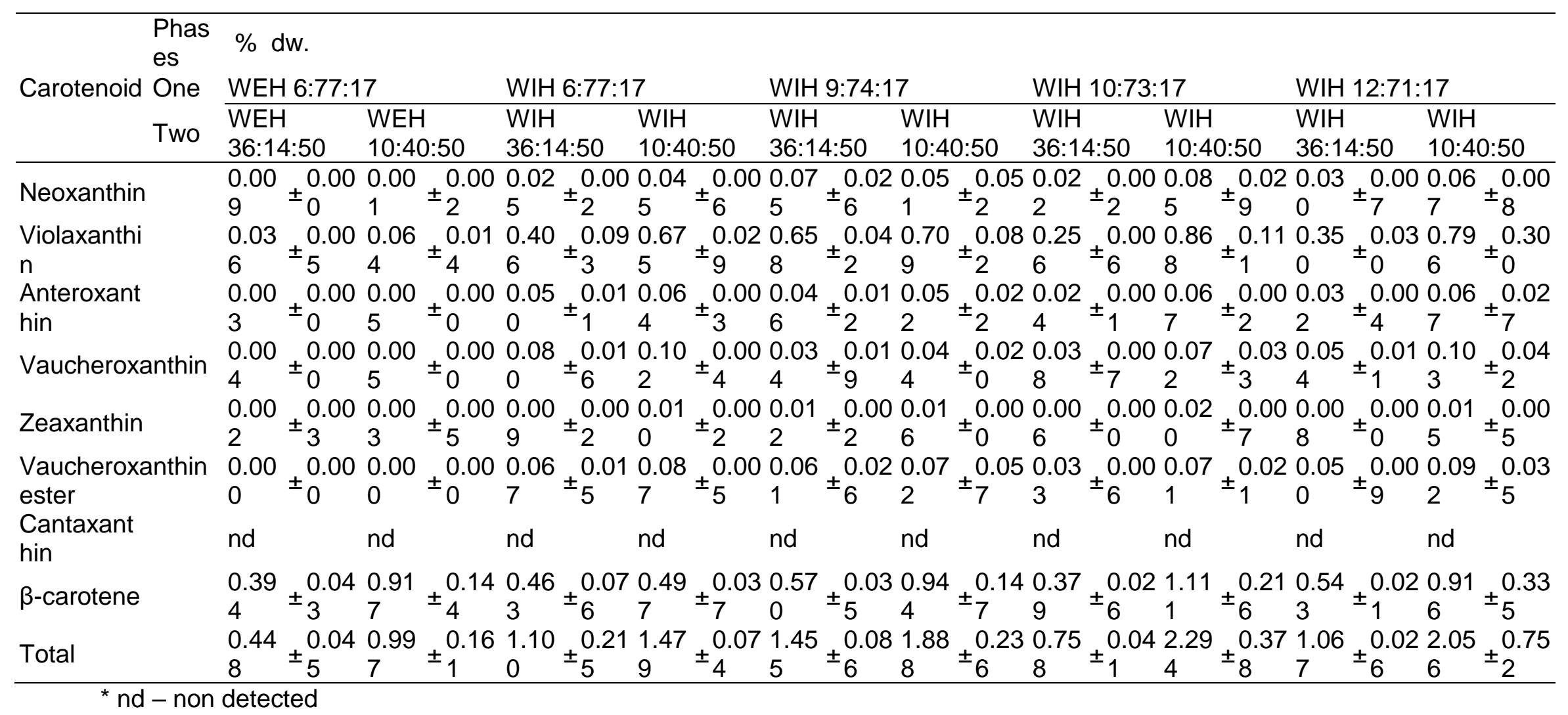


Table 5 - Fatty acid content in dry weight (\% d.w.) mean \pm SD of fatty acid composition of Nannochloropsis gaditana batch 1 of the extraction in small scale.

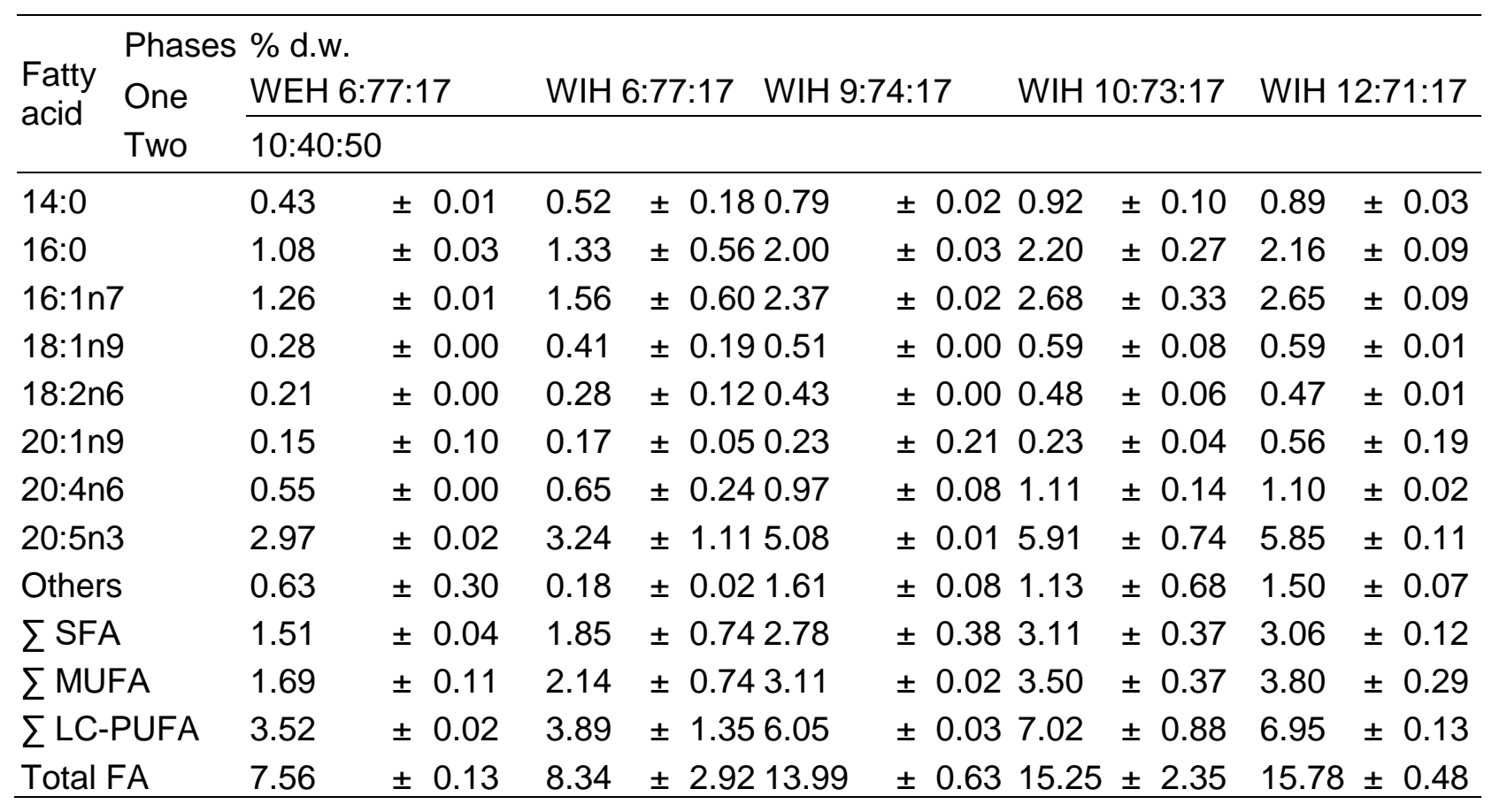




\section{STANDARD METHOD}

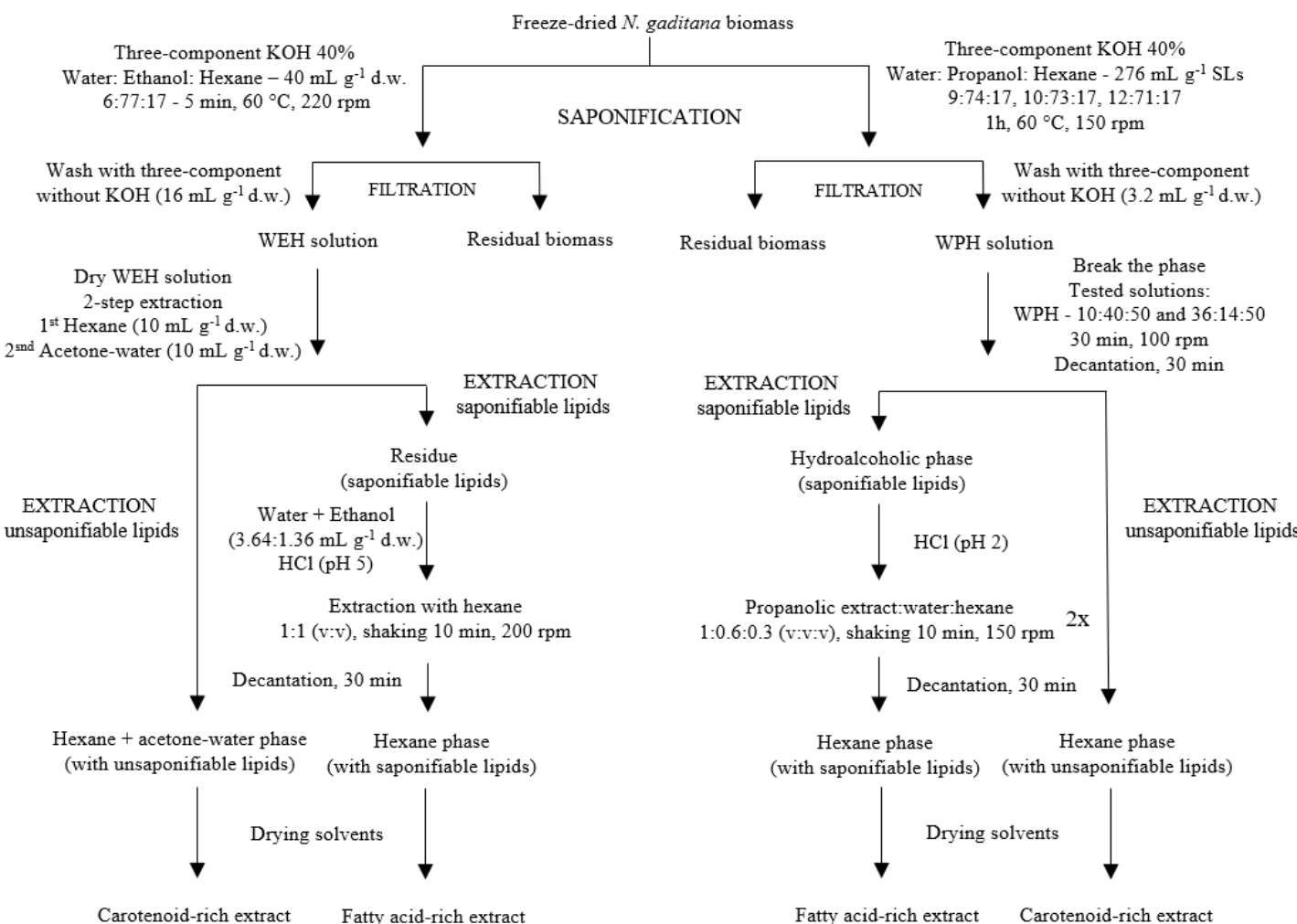




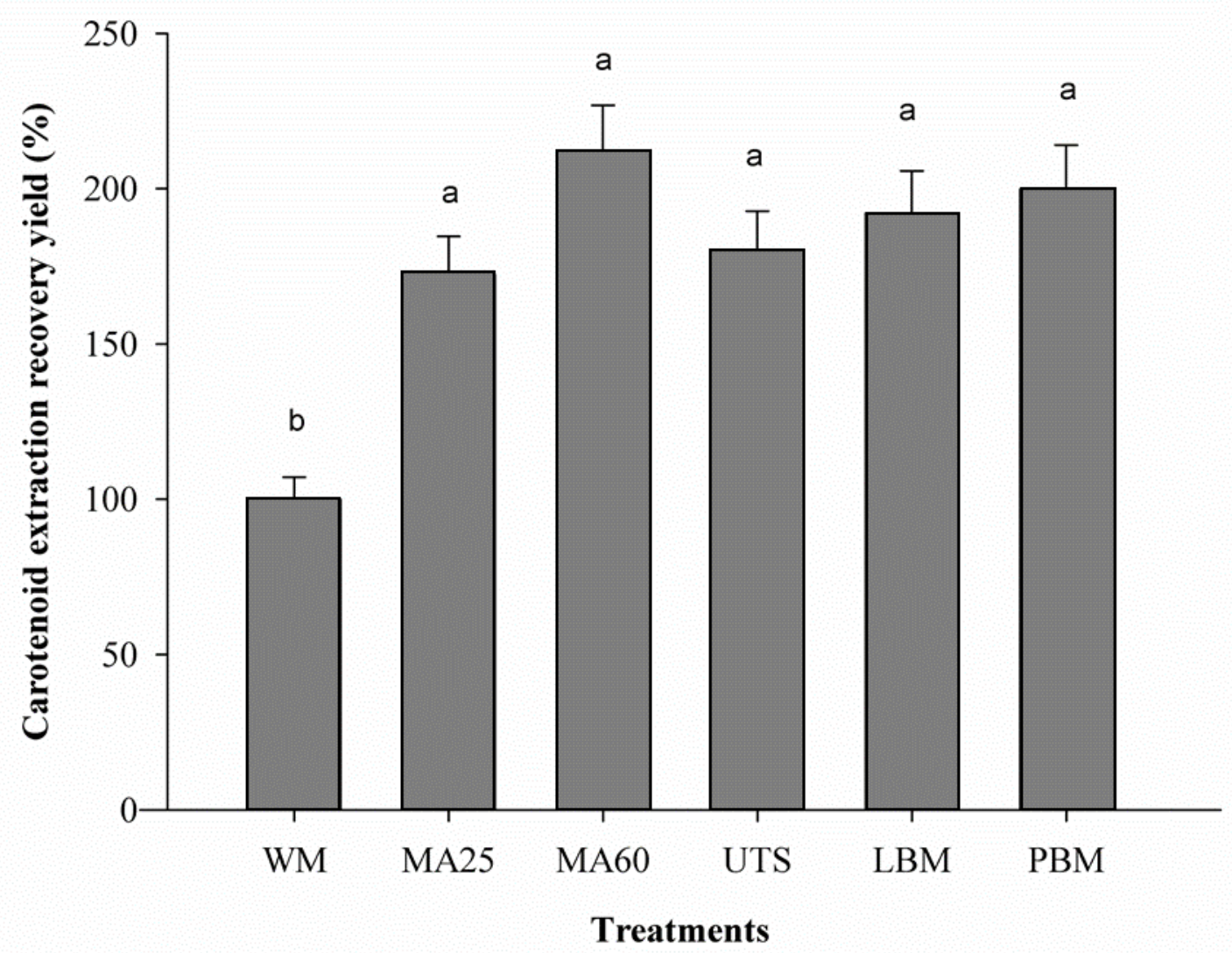




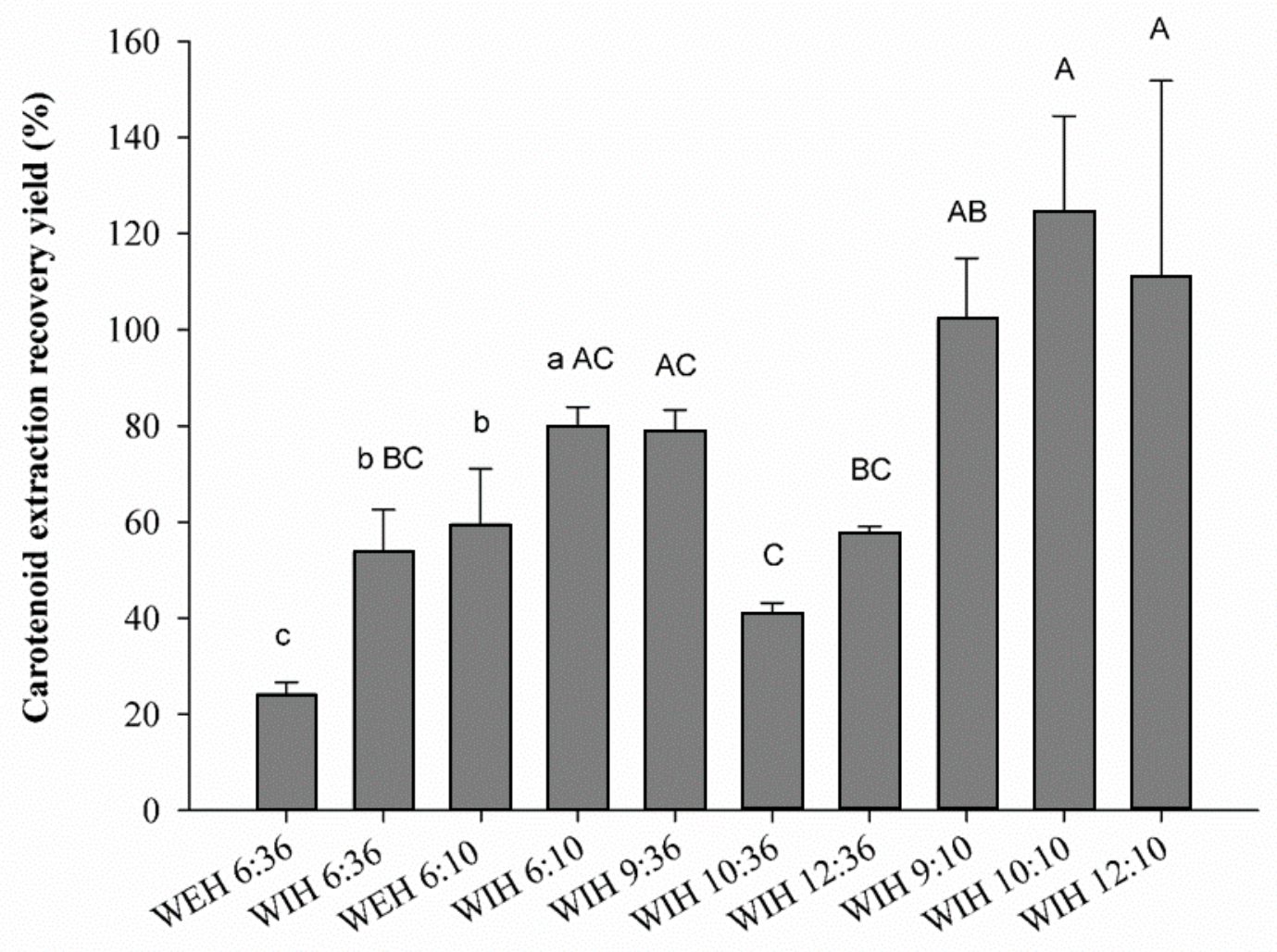

Treatments 


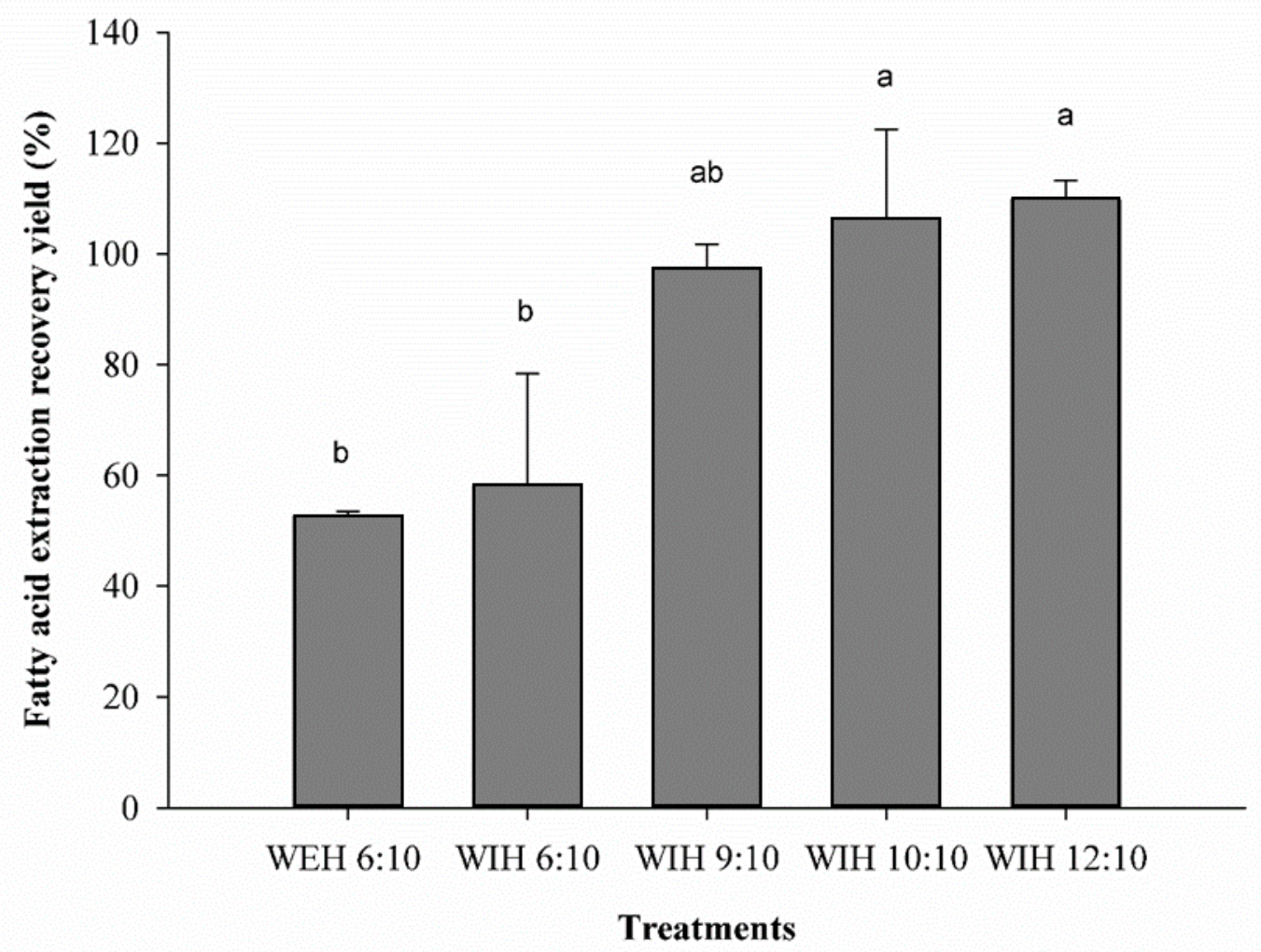



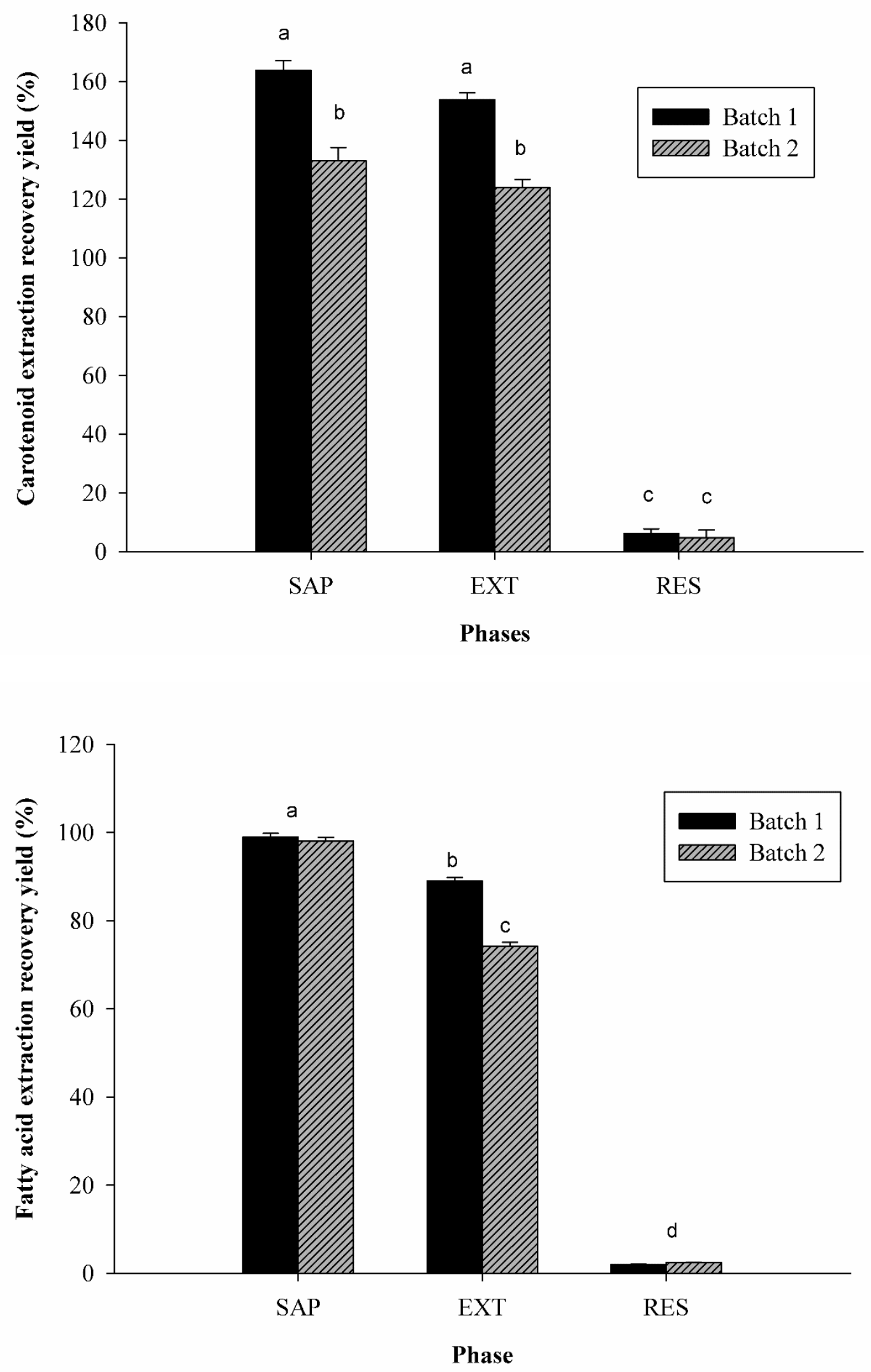